\title{
Identification of two novel mutations in the SLCO2AI prostaglandin transporter gene in a Chinese patient with primary hypertrophic osteoarthropathy
}

\author{
TING GUO $^{1 *}$, KAI YANG ${ }^{2 *}$, LV LIU $^{1}$, ZHI-PING TAN ${ }^{3}$ and HONG LUO ${ }^{1}$ \\ ${ }^{1}$ Department of Respiratory Medicine, The Second Xiangya Hospital of Central South University; ${ }^{2}$ Xiangya School \\ of Medicine, Central South University; ${ }^{3}$ Clinical Center for Gene Diagnosis and Therapy, State Key Laboratory of \\ Medical Genetics, The Second Xiangya Hospital of Central South University, Changsha, Hunan 410011, P.R. China
}

Received January 27, 2016; Accepted January 16, 2017

DOI: $10.3892 / \mathrm{mmr} .2017 .6391$

\begin{abstract}
Primary hypertrophic osteoarthropathy (PHO), which is a rare multi-organic disease characterized by digital clubbing, pachydermia and periosteal reaction, typically begins during childhood or adolescence and progresses gradually over years prior to disease stabilization. To date, only two genes have been reported to be associated with PHO, 15-hydroxyprostaglandin dehydrogenase and solute carrier organic anion transporter family, member 2A1 (SLCO2A1). However, the pathogenesis and the functions of the underlying genes remain to be fully elucidated. In the present study, a 20-year-old Chinese patient with PHO was investigated using sequence analysis of PHO genes and bioinformatics analysis. A novel, compound heterozygous mutation in the SLCO2AI gene was identified, which contained two novel mutations: c.349delC (p.L117SfsX56) in exon 3 and c.1286A>G (p.Y429C) in exon 9. These two novel genotypes in PHO are the first, to the best of our knowledge, to be reported in PHO. This finding expands the mutation spectrum of $\mathrm{PHO}$, which contributes to improving genetic diagnosis and future genetic counseling, and provides clues to the phenotype-genotype associations.
\end{abstract}

\section{Introduction}

Primary hypertrophic osteoarthropathy (PHO; MIM 259100 and 614441), also known as pachydermoperiostosis, is a rare

Correspondence to: Professor Hong Luo, Department of Respiratory Medicine, The Second Xiangya Hospital of Central South University, 139 RenMin Middle Road, Changsha, Hunan 410011, P.R. China

E-mail: luohong1003@163.com

${ }^{*}$ Contributed equally

Key words: primary hypertrophic osteoarthropathy, solute carrier organic anion transporter family member $2 \mathrm{~A} 1$, genetic diagnosis, phenotype multi-organic, disease primarily characterized by digital clubbing, periostosis and pachydermia (1). The manifestations usually begin at puberty and progress gradually over years prior to stabilization of the disease. As the primary form of hypertrophic osteoarthropathy, $\mathrm{PHO}$ is distinct from the more common secondary hypertrophic osteoarthropathy, which is always associated with an underlying cause, for example pulmonary or cardiac disease. In terms of the inheritance of $\mathrm{PHO}$, autosomal dominant with incomplete penetrance and recessive inheritance have previously been confirmed $(2,3)$. However, the precise incidence and prevalence remain to be elucidated (4).

PHO is a genetically heterogeneous disease. To date, two genes have been reported to be associated with PHO $(5,6)$. In 2008, 15-hydroxyprostaglandin dehydrogenase (HPGD; MIM 601688), which encodes 15-PGDH, was identified as the primary causative factor of PHO. Zhang et al (6) demonstrated another pathogenic gene responsible for $\mathrm{PHO}$, solute carrier organic anion transporter family, member 2A1 (SLCO2A1; MIM 601460), which is a gene encoding the prostaglandin transporter. It has been suggested that the two genes, $H P G D$ and $S L C O 2 A 1$, are involved in the degradation of prostaglandin E2 (PGE2). The degradation of PGE2 involves two steps (7): Selective uptake across the plasma membrane by SLCO2Al and degradation inside the cell by $H P G D$. Considering these molecular findings, $\mathrm{PHO}$ has been categorized into two types (1): i) hypertrophic osteoarthropathy, primary, autosomal recessive, type 1 (PHOAR1; MIM 259100) due to HPGD deficiency; and ii) hypertrophic osteoarthropathy, primary, autosomal recessive, type 2 (PHOAR2; MIM 614441) due to $S L C O 2 A 1$ deficiency.

When examining a patient with digital clubbing, it is important to differentiate between secondary hypertrophic osteoarthropathy due to pulmonary disease or other conditions, which is more common, and PHO, which is less common. However, several clinical examinations are required to eliminate secondary causes, which is an expensive and time-consuming process. In addition, as PHO is clinically rare and the disease remains to be fully understood, it can lead to a missed diagnosis or misdiagnosis and consequent suboptimal treatment. At present, the identification of SLCO2A1 


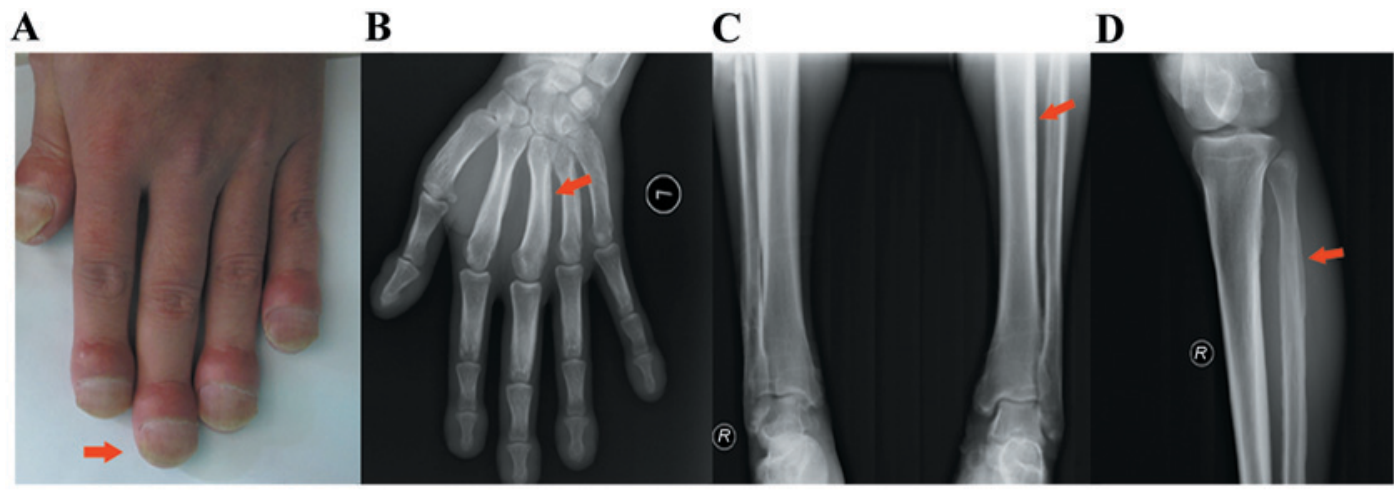

Figure 1. Clinical features of primary hypertrophic osteoarthropathy. (A) Marked digital clubbing. (B-D) Periosteal overgrowth of the long bones of the affected individual. The red arrows indicate areas of marked periosteal hyperostosis. (B) Periosteal hyperostosis of the metacarpus and phalange. (C) Periosteal hyperostosis of the tibia. (D) Periosteal hyperostosis of the fibula.

or HPGD deficiency in patients with PHO has become an efficient approach to assist in clinical diagnoses. However, the genotype/phenotype associations have not been observed, and the clinical phenotype and mutational spectrum of these pathogenic genes require further investigation in order to fully understand this disease. The present study reported on a 20-year-old Chinese patient with PHO. The study identified two novel mutations (c.349delC/p.L117SfsX56 and c.1286A $>$ G/p.Y429C) in the SLCO2Al gene in PHO disease. To the best of our knowledge, these mutations have not been reported in a previous study, or presented in the Single Nucleotide Polymorphism Database (dbSNP) (http://www. ncbi.nlm.nih.gov/projects/SNP) or the Human Gene Mutation Database (http://www.hgmd.cf.ac.uk/ac/index.php).

\section{Materials and methods}

Clinical findings. The protocol for the present study was approved by the Review Board of The Second Xiangya Hospital of the Central South University (Changsha, China), and the participants involved provided informed written consent. All experiments were performed in accordance with relevant guidelines and regulations. A 20-year-old man was born to healthy non-consanguineous parents. Apparently healthy during his childhood, he reported that at 18 years of age, he noticed progressive enlargement of the terminal phalanges and pachydermia (Fig. 1A). After 2 years, he gradually presented with joint discomfort and action inconvenience. He denied any gastrointestinal discomfort, blepharitis, seborrhea, or hyperhidrosis. Radiography revealed periosteal overgrowth of the tibiofibular joint and a region of the metacarpus (Fig. 1B-D). Physical examination revealed no other secondary hypertrophic osteoarthropathy, including congenital heart disease or lung abnormities. All other laboratory investigations were within normal limits, including complete blood count, liver function test, renal profile, thyroid stimulating hormone and growth hormone. The patient had no siblings, and his parents were healthy without the features described above.

Sequence analysis of associated genes. Informed consent was obtained from the patient and from 200 healthy volunteers (19-54 years old; 108 male, 92 female; recruited from the physical examination center of the Second Xiangya Hospital of Central South Hospital, Changsha, China) prior to blood sampling and DNA analysis. The DNA was extracted from peripheral white blood cells using conventional methods. The DNA sequences of SLCO2Al and HPGD were obtained from the Ensembl online database (http://asia.ensembl. org/index.html; Ensembl nos. ENSG00000174640 and ENSG00000164120, respectively). Primers for polymerase chain reaction (PCR) were designed using IDT software (http://www.idtdna.com/site) and the sequences are listed in Table I. The entire coding regions, including the flanking intronic sequences of SLCO2Al and HPGD, were amplified by PCR. Patient DNA samples were amplified in $25 \mu \mathrm{l}$ reactions using 2X Power Taq PCR MasterMix (12.5 $\mu$ l; Bioteke Corporation, Beijing, China), nuclease-free water (11 $\mu 1)$, $10 \mathrm{pmol} / \mathrm{ul}$ forward and reverse primers $(0.5 \mu \mathrm{l}$ each $)$, and $100 \mathrm{ng} / \mu 1$ template $(0.5 \mu \mathrm{l})$. Thermocycling conditions were as follows: Initial denaturation at $94^{\circ} \mathrm{C}$ for $5 \mathrm{~min}, 35$ cycles of denaturation at $94^{\circ} \mathrm{C}(30 \mathrm{sec})$, annealing temperature $(30 \mathrm{sec})$ (Table I), extension at $72^{\circ} \mathrm{C}(1 \mathrm{~min})$, and final extension at $72^{\circ} \mathrm{C}$ (7 min). Sequences of the PCR products were determined using the ABI 3100 genetic analyzer (Thermo Fisher Scientific, Inc., Waltham, MA, USA) as previously described (8). To assess the damaging effects of variants in silico, the online databases, Polyphen2 (http://genetics.bwh.harvard.edu/pph2) (9) and MutationTaster (www.mutationtaster.org) (10), were used to predict the possible effects of variants.

\section{Results}

In the present study the SLCO2A1 and HPGD mutations were screened for in the patient and his parents using PCR followed by direct sequence analysis (Fig. 2). Sequencing revealed compound heterozygous mutations in the SLCO2AI gene, which consisted of a novel frameshift deletion, p.L117SfsX56 (c.349 delC), in exon 3 (Fig. 2A) and a novel missense mutation, p.Y429C (c.1286A $>$ G), in exon 9 (Fig. 2B). The p.L117SfsX56 mutation resulted in a frameshift at amino acid position 117 and the introduction of a premature stop codon after 56 amino acid residues, and was predicted to be disease-causing by MutationTaster (www.mutationtaster. org), with a probability value of 1 . The p.Y429C mutation was identified as non-conservative, affected evolutionarily conserved amino acids in diverse species (Fig. 2I) and 
Table I. Primer sequences for polymerase chain reaction.

\begin{tabular}{|c|c|c|}
\hline Gene & Primer sequence $\left(5^{\prime} \rightarrow 3^{\prime}\right)$ & Annealing temperature $\left({ }^{\circ} \mathrm{C}\right)$ \\
\hline \multirow[t]{2}{*}{ SLCO2A1 (1) } & F: ACCCTCATATCCCAGCCTTC & 59.8 \\
\hline & R: TGTCCGAGTAAGCGGTAAGC & 59.8 \\
\hline \multirow[t]{2}{*}{ SLCO2A1 (2) } & F: CACTGGGCCACATATCACAG & 59.8 \\
\hline & R: CTGTTACCCGGCAGAAAGAG & 59.8 \\
\hline \multirow[t]{2}{*}{ SLCO2A1 (3) } & F: GGAGATGGAGACCCAGAAGG & 61.9 \\
\hline & R: GCACACTTTCCTGAACAAACC & 58.0 \\
\hline \multirow[t]{2}{*}{ SLCO2A1 (4) } & F: CAGGAACCATGTCCCATTTG & 57.8 \\
\hline & R: ACACAGCTGGGAGGTAATGG & 59.8 \\
\hline \multirow[t]{2}{*}{ SLCO2A1 (5) } & F: ACAGGTGTGGGCTTATCAGG & 59.8 \\
\hline & R: CAGCAGCTTGTTCCTCACAG & 59.8 \\
\hline \multirow[t]{2}{*}{ SLCO2A1 (6) } & F: CCTCTGGGAAGACCAATAGC & 59.8 \\
\hline & R: TGGAGGTCTCCTGATCCTTG & 59.8 \\
\hline \multirow[t]{2}{*}{ SLCO2A1 (7) } & F: GGAAATGCAGGTGCTGTTTG & 57.8 \\
\hline & R: TCTGCTCCTACTGTCCCTTAC & 60.0 \\
\hline \multirow[t]{2}{*}{ SLCO2A1 (8) } & F: CCCTGTGGTGTTGTGTGC & 59.6 \\
\hline & R: CTGACTGGAAGGACAGGAG & 59.7 \\
\hline \multirow[t]{2}{*}{ SLCO2A1 (9) } & F: GCCTGGCAAGCAGTAAATG & 57.6 \\
\hline & R: TGCTTGAACCTGGGAGAATC & 57.8 \\
\hline \multirow[t]{2}{*}{ SLCO2A1 (10) } & F: AAATGGAGAGATGCCGTGAC & 57.8 \\
\hline & R: CCCAGGGTAGGGAGGTAGAG & 64.0 \\
\hline \multirow[t]{2}{*}{ SLCO2A1 (11) } & F: TTGCCCAAACAGTGACAGAG & 57.8 \\
\hline & R: CCTGCAATGAGGAGCTCAG & 59.7 \\
\hline \multirow[t]{2}{*}{ SLCO2A1 (12) } & F: TAGAGCATTCAGCCCAGGTG & 59.8 \\
\hline & R: CCTCAAGCAATCTGGGAAAC & 57.8 \\
\hline \multirow[t]{2}{*}{ SLCO2A1 (13) } & F: GCCCGTGTATCTCCACTCTG & 61.9 \\
\hline & R: TGGCCCTTCATGTTCTCTTC & 57.8 \\
\hline \multirow[t]{2}{*}{ SLCO2A1 (14) } & F: CCTGCTTCCCTACAGCTTTG & 59.8 \\
\hline & R: GGGTACACAGTGGCCCTTAG & 61.9 \\
\hline \multirow[t]{2}{*}{ HPGD (1) } & F: GCTGGCTTGACAGTTTCCTC & 59.8 \\
\hline & R: AGTCTCGGAGTGTGTGGGC & 61.9 \\
\hline \multirow[t]{2}{*}{ HPGD (2) } & F: GTGTTTATTGTTTGTCCGTCTA & 54.5 \\
\hline & R: CAGTCTTGCCTTTCTTTCG & 55.4 \\
\hline \multirow[t]{2}{*}{ HPGD (3) } & F: CCTCTCATGGCATAGGACATG & 60.0 \\
\hline & R: GTTTCCATGACTCCAAGAACC & 58.0 \\
\hline \multirow[t]{2}{*}{ HPGD (4) } & F: CCACAATGATTAGGCAAAC & 53.2 \\
\hline & R: AAGCCACAAGTTAAATTAAGAG & 52.6 \\
\hline \multirow[t]{2}{*}{ HPGD (5) } & F: AAATTCTGGACGACACGG & 55.0 \\
\hline & R: TTCCACCTTTCATCCAAGT & 53.2 \\
\hline \multirow[t]{2}{*}{ HPGD (6) } & F: TTGTTACATAGCTGGGAG & 52.7 \\
\hline & R: ATAATGCTTTGCTTCATC & 48.2 \\
\hline \multirow[t]{2}{*}{ HPGD (7) } & F: ATGCCTCATTCTTTCGTT & 50.5 \\
\hline & R: TAGCCTTTGGTCCACATC & 55.0 \\
\hline
\end{tabular}

F, forward; HPGD, 15-hydroxyprostaglandin dehydrogenase; R, reverse; SLCO2A1, solute carrier organic anion transporter family, member $2 \mathrm{~A} 1$.

was predicted in silico by all bioinformatics tools used to be of pathogenic relevance (PolyPhen-2 score, 0.999; MutationTaster score, 0.999). Based on the DNA sequence analysis, the p.L117SfsX56 and p.Y429C mutations were found to be inherited from the patient's unaffected father and mother, respectively, and were not found in the cohort of 200 control individuals (Fig. 2C-H) (8). Neither of these mutations has been reported in a previous study, or was present in the dbSNP or Human Gene Mutation Database (http://www. hgmd.cf.ac.uk/ac/index.php). 


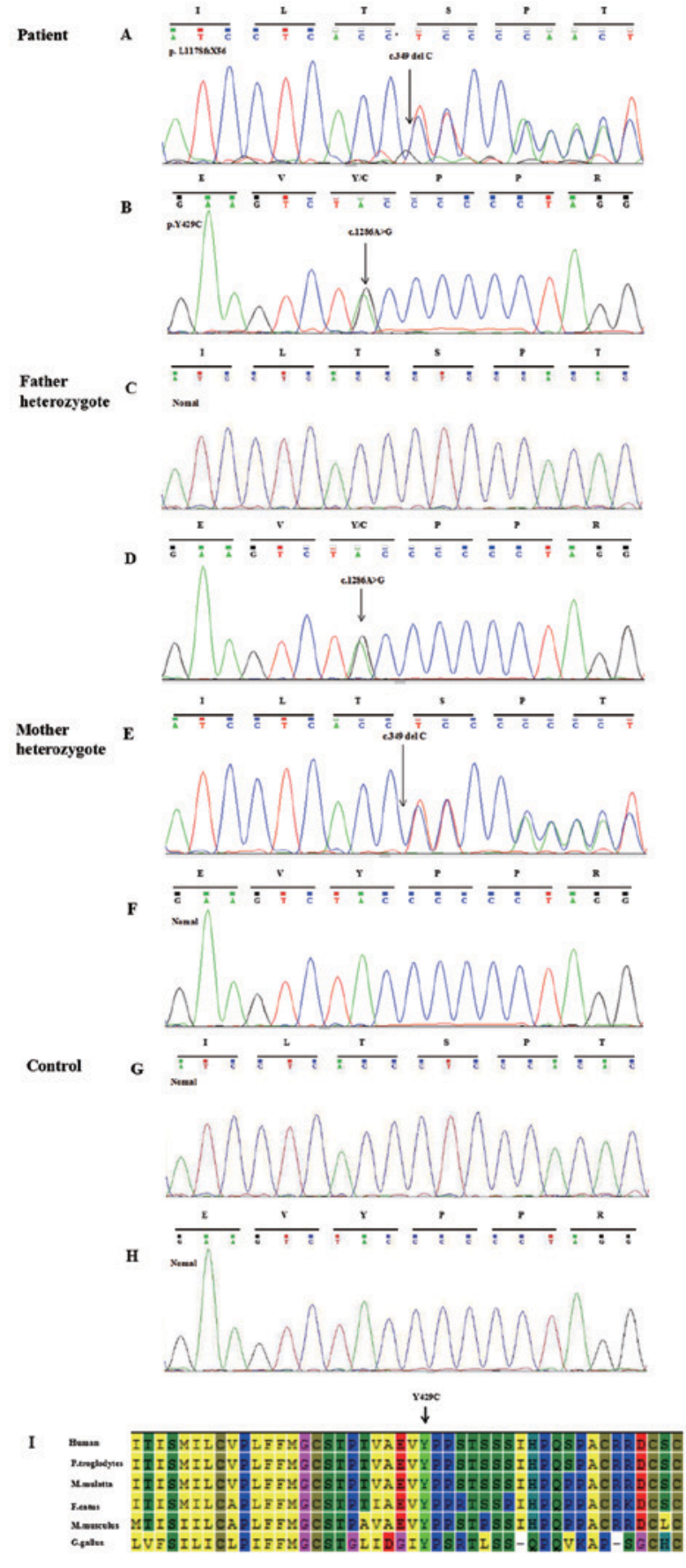

Figure 2. Genetic analysis of the solute carrier organic anion transporter family, member $2 \mathrm{~A} 1$ gene in the patient, his parents and a control. (A and B) A compound heterozygous mutation in the patient: (A) c.349delC (p.L117SfsX56) in exon 3; and (B) c.1286A $>$ G (p.Y429C) in exon 9. (C and D) Heterozygote, c.1286A>G (p.Y429C), was identified in the patient's father. (E and F) Heterozygote, c.349delC (p.L117SfsX56), was identified in the patient's mother. ( $\mathrm{G}$ and $\mathrm{H}$ ) No mutations were observed in the control (I) p.Y429C was revealed to be highly evolutionarily conserved in diverse species. M. mulatta, Macaca mulatta; F. catus, Felis catus; M. musculus, Mus musculus; G. gallus, Gallus gallus; P. troglodytes, Pan troglodytes.

\section{Discussion}

As is already known, the central feature in the pathogenesis of PHO is the failure of PGE2 degradation. However, a number of genes are directly involved in the biosynthesis and signaling pathway of the PGE2, including HPGD, prostaglandin-endoperoxidase synthase (PTGS) 1, PTGS2, prostaglandin E synthase (PTGES), PTGES2, PTGES3, PTGER1, prostaglandin E receptor (PTGER) 2, PTGER3, PTGER4, SLCO2A1, SLCO3A1, SLCO4A1, prostaglandin reductase (PTGR) 1 and PTGR2 (7). Among these, Zhang et al (6) first demonstrated in 2012 that SLCO2Al is a PHO-pathogenic gene. The SLCO2Al gene is located on chromosome $3 \mathrm{q} 21$ and organized into 14 exons, which encode a 643 amino acid, 12-transmembrane-domain organic anion cell-surface transporter.

To date, 45 different SLCO2Al mutations, including 30 missense/nonsense mutations, eight splice sites and seven indels, have been identified in patients with PHO. As shown in Fig. 3, the eight splice sites were predominantly concentrated between exons 7 and 8 , and the majority of other mutations were positioned on exons 4, 6 and 9. In the present study, two novel mutations were identified in the SLCO2Al gene in PHO disease, comprising c.349delC/p.L117SfsX56 in exon 3 and c.1286A $>$ G/p.Y429C in exon 9. One of these mutations, c.1286A $>\mathrm{G}$ (p.Y429C), was identified within the region of a previously reported deletion (11), c.1279_1290del12 (p.E427_ P430del). Sasaki et al (11) reported that the amino acid sequence containing the p.E427_P430del mutation is located in the extracellular region between the 9th and 10th transmembrane domains, and this mutation may have a less severe effect on PG transport activity (12), which may be consistent with the faint pachydermia. The patient examined in the present study was consistent with the report by Sasaki et al (11), describing an incomplete form of PHO, including finger clubbing and periostosis, but lacking cutis verticis gyrate (CVG).

With the exception of the three major phenotypes of clubbing, periostosis and pachydermia, additional symptoms, including sebaceous hyperplasia, hyperhidrosis, blepharoptosis and arthropathy have also been reported $(13,14)$. It has also been reported that, compared with the clinical spectrum of patients affected with $H P G D$ mutations, the clinical manifestations in patients with SLCO2Al mutations emerge later, beginning with clubbing of the distal phalanges during puberty and pachydermia shortly following puberty. However, the degree of arthritis, joint involvement and pachydermia in patients affected by $S L C O 2 A 1$ mutations appear to be more pronounced, compared with that in individuals with homozygous or compound heterozygous HPGD mutations (7). In the present study, the patient gradually presented with joint discomfort and affected activity, however, hyperhidrosis, sebaceous hyperplasia and blepharoptosis were not present. Hyperhidrosis, sebaceous hyperplasia and blepharoptosis can all fall under skin symptoms. The pathogenetic mechanism of skin symptoms involve epidermal and sebaceous gland hyperplasia, and dermis hypoplasia, and these cell proliferation differences between the dermis and epidermis determine the magnitude of the affected skin in PHO $(11,15,16)$. Due to the delayed clinical manifestations in the patient with SLCO2AI mutations in the present study, it is not possible to exclude the possibility of slowly emerging skin symptoms following puberty. Thus, close regular follow-up is required.

A previous study has reported that $S L C O 2 A 1$-deficient patients may present with chronic anemia secondary to hypocellular myelofibrosis (17). The incidence of anemia in Caucasian patients with PHO was found to be almost 50\% (10/19). The 


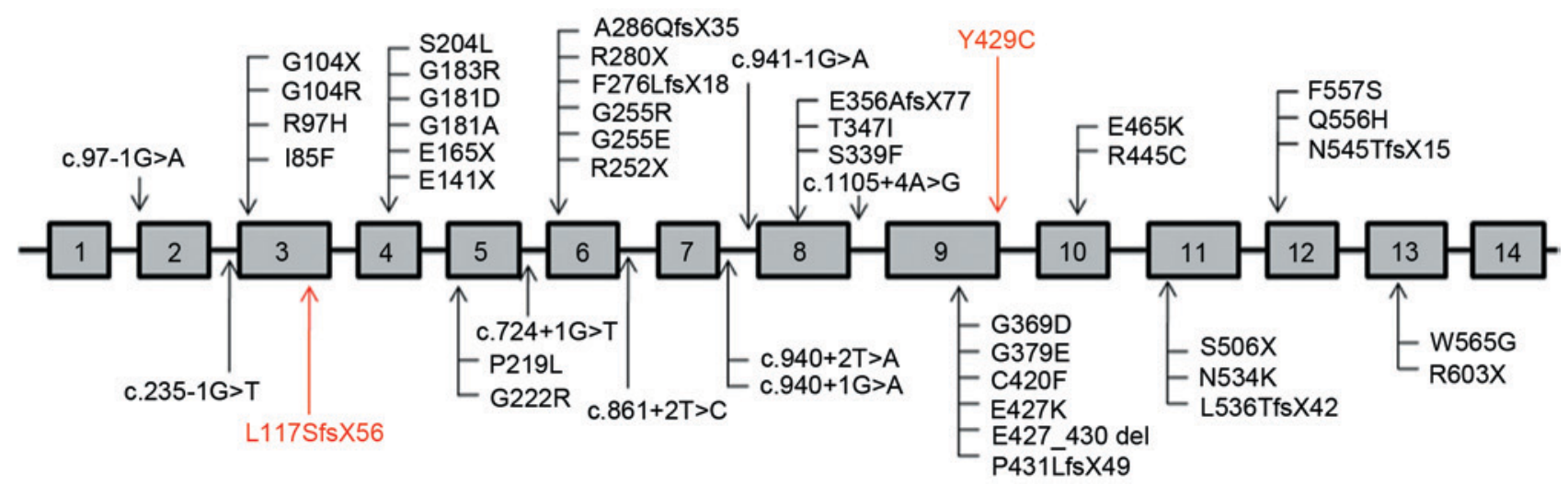

Figure 3. Summary of mutations identified in the solute carrier organic anion transporter family, member $2 \mathrm{~A} 1$ gene in primary hypertrophic osteoarthropathy. Black arrows indicate previously reported mutations. The red arrows indicate the novel mutation identified in the present study.

possible mechanism may be associated with complex pleiotropic effects of PGE2 on multilineage and lineage-restricted hematopoietic progenitor cells (18), however, in the present study and other studies $(1,6,19)$, the incidence of anemia in Chinese patients is low. This data may reflect ethnic differences.

Another specific symptom reported is gastrointestinal discomfort. Common gastrointestinal involvement includes chronic gastritis, hypertrophic gastropathy, peptic ulcer, Crohn's disease and watery diarrhea (1). The patient in the present study denied any gastrointestinal symptoms. Thus, gastroscopy, gastric mucosa biopsy and associated gastrointestinal system examinations were not performed. However, considering hypertrophic gastropathy, this disease often lacks typical symptoms and the majority of patients with hypertrophic gastropathy often show no symptoms, despite the presence of gastric mucosal lesions and hypertrophy on gastroscopy observation. Thus, additional specimen collection and analyses is required to analyze the incidence and prevalence of hypertrophic gastropathy in PHO.

In conclusion, the present study identified two novel mutations (c.349delC/p.L117SfsX56 and c.1286A>G/p.Y429C) in SLCO2A1 in a patient with PHO. These mutations of SLCO2AI (p.L117SfsX56 and p.Y429C) are the first, to the best of our knowledge, to be reported in $\mathrm{PHO}$, and the finding provides clues to phenotype-genotype associations. In addition, the findings obtained in the present study are consistent with those of other studies and suggests that p.Y429C may have a less severe effect on PG transport activity, and may be consistent with the faint pachydermia. The results of the present study expand the mutant spectrum of $\mathrm{PHO}$, which contributes to a more rapid genetic diagnosis, and interpretation of genetic information for prenatal diagnosis and genetic counseling.

\section{Acknowledgements}

The authors would like to thank the State Key Laboratory of Medical Genetics of China for their technical assistance. This study was supported by the National Natural Science Foundation of China (grant nos. 81470202 and 81570288), the Natural Science Foundation of Hunan Province, China (grant nos. 13JJ3020 and 2015JC3032), the Scientific and Technological Project of Changsha City (grant no. K1406020-31) and the National Key Clinical Specialty Construction Projects of China.

\section{References}

1. Zhang Z, He JW, Fu WZ, Zhang CQ and Zhang ZL: Mutations in the SLCO2A1 gene and primary hypertrophic osteoarthropathy: A clinical and biochemical characterization. J Clin Endocrinol Metab 98: E923-E933, 2013.

2. Oikarinen A, Palatsi R, Kylmäniemi M, Keski-Oja J, Risteli J and Kallioinen M: Pachydermoperiostosis: Analysis of the connective tissue abnormality in one family. J Am Acad Dermatol 31: 947-953, 1994.

3. Rimoin DL: Pachydermoperiostosis (idiopathic clubbing and periostosis): Genetic and physiologic considerations. N Engl J Med 272: 923-931, 1965.

4. Jajic I: Epidemiology of hypertrophic osteoarthropathy. Clin Exp Rheumatol 10 (Suppl 7): S13, 1992.

5. Uppal S, Diggle CP, Carr IM, Fishwick CW, Ahmed M, Ibrahim GH, Helliwell PS, Latos-Bieleńska A, Phillips SE, Markham AF, et al: Mutations in 15-hydroxyprostaglandin dehydrogenase cause primary hypertrophic osteoarthropathy. Nat Genet 40: 789-793, 2008.

6. Zhang Z, Xia W, He J, Zhang Z, Ke Y, Yue H, Wang C, Zhang H, $\mathrm{Gu} \mathrm{J}, \mathrm{Hu} \mathrm{W}$, et al: Exome sequencing identifies SLCO2A1 mutations as a cause of primary hypertrophic osteoarthropathy. Am J Hum Genet 90: 125-132, 2012.

7. Seifert W, Kühnisch J, Tüysüz B, Specker C, Brouwers A and Horn D: Mutations in the prostaglandin transporter encoding gene SLCO2A1 cause primary hypertrophic osteoarthropathy and isolated digital clubbing. Hum Mutat 33: 660-664, 2012.

8. Tan ZP, Huang C, Xu ZB, Yang JF and Yang YF: Novel ZFPM2/FOG2 variants in patients with double outlet right ventricle. Clin Genet 82: 466-471, 2012.

9. Sunyaev S, Ramensky V and Bork P: Towards a structural basis of human non-synonymous single nucleotide polymorphisms. Trends Genet 16: 198-200, 2000.

10. Schwarz JM, Rödelsperger C, Schuelke M and Seelow D: Mutation taster evaluates disease-causing potential of sequence alterations. Nat Methods 7: 575-576, 2010.

11. Sasaki T, Niizeki H, Shimizu A, Shiohama A, Hirakiyama A, Okuyama T, Seki A, Kabashima K, Otsuka A, Ishiko A, et al: Identification of mutations in the prostaglandin transporter gene SLCO2A1 and its phenotype-genotype correlation in Japanese patients with pachydermoperiostosis. J Dermatol Sci 68: 36-44, 2012.

12. Minakawa S, Kaneko T, Niizeki H, Mizukami H, Saito Y, Nigawara T, Kurose R, Nakabayashi K, Kabashima K and Sawamura D: Case of pachydermoperiostosis with solute carrier organic anion transporter family, member 2A1 (SLCO2A1) mutations. J Dermatol 42: 908-910, 2015.

13. Madruga Dias JA, Rosa RS, Perpétuo I, Rodrigues AM, Janeiro A, Costa MM, Gaião L, Pereira da Silva JA, Fonseca JE and Miltenberger-Miltenyi G: Pachydermoperiostosis in an African patient caused by a Chinese/Japanese SLCO2A1 mutation-case report and review of literature. Semin Arthritis Rheum 43: 566-569, 2014.

14. Zhang Z, He JW, Fu WZ, Zhang CQ and Zhang ZL: Two novel mutations in the SLCO2A1 gene in a Chinese patient with primary hypertrophic osteoarthropathy. Gene 534: 421-423, 2014. 
15. Neufang G, Furstenberger G, Heidt M, Marks F and Müller-Decker K: Abnormal differentiation of epidermis in transgenic mice constitutively expressing cyclooxygenase-2 in skin. Proc Natl Acad Sci USA 98: 7629-7634, 2001

16. Weinberg E, Topaz M, Dard M, Lyngstadaas P, Nemcovsky C and Weinreb M: Differential effects of prostaglandin E(2) and enamel matrix derivative on the proliferation of human gingival and dermal fibroblasts and gingival keratinocytes. J Periodontal Res 45: 731-740, 2010.

17. Diggle CP, Parry DA, Logan CV, Laissue P, Rivera C, Restrepo CM, Fonseca DJ, Morgan JE, Allanore Y, Fontenay M, et al: Prostaglandin transporter mutations cause pachydermoperiostosis with myelofibrosis. Hum Mutat 33: 1175-1181, 2012.
18. North TE, Goessling W, Walkley CR, Lengerke C, Kopani KR, Lord AM, Weber GJ, Bowman TV, Jang IH, Grosser T, et al: Prostaglandin E2 regulates vertebrate haematopoietic stem cell homeostasis. Nature 447: 1007-1011, 2007.

19. Zhang Z, He JW, Fu WZ, Zhang CQ and Zhang ZL: A novel mutation in the SLCO2A1 gene in a Chinese family with primary hypertrophic osteoarthropathy. Gene 521: 191-194, 2013. 